Bol. Soc. Bot. México 49:61.69(1989)

\title{
El almacenamiento de semillas en la conservación de especies vegetales. Problemas y aplicaciones
}

\author{
CARlos VAzQueZ-Yanes y Jorge R. TOLEDO ${ }^{1}$
}

\begin{abstract}
RESUMEN. La semillas han sido clasificadas en ortodoxas y recalcitrantes de acuerdo con las posibilidades de preservar su viabilidad en condiciones de almacenamiento. Las primeras pueden deshidratarse y almacenarse a baja temperatura, en tanto que las segundas no sobreviven a estos tratamientos. Es relativamente fácil utilizar el almacenamiento de semillas como una forma de conservación del germoplasma cuando se manejan semillas ortodoxas; en cambio, sólo a través de la profundización en el conocimiento de la estructura y fisiología de las semillas recalcitrantes, será posible algún día almacenarlas por largo tiempo con éxito.
\end{abstract}

ABSTRACT. Seeds have been classified in two groups: ortodox type and recalcitrant type according to their storage properties. The first type of seeds can be dehidratated and stored at low temperature. The second type, they do not survive those treatments. It is relatively easy to store seeds as a way of preservation of germoplasm of endangered species in the case of ortodox seeds. On the other hand, much more research is needed to deal with recalcitrant seeds for the purpose of conservation.

\section{INTRODUCCION.}

Una de las formas más efectivas de preservar el germoplasma de especies vegetales silvestres y de promover la repoblación del medio natural del que proceden es mediante el almacenamiento de semillas en condiciones controladas, que pemitan prolongar la duración de su viabilidad. Existen en el mundo algunos almacenes o bancos de semillas que tienen esta finalidad; sin embargo, el esfuerzo realizado hasta el momento ha sido

${ }^{1}$ Centro de Ecología. Universidad Nacional Autónoma de México, Apdo. Postal 70-275, Delegación Coyoacán, 04510 México, D. F.

Vazquez-Yanes C, Toledo JR. 1989. El almacenamiento de semillas en la conservación de especies vegetales. Problemas y aplicaciones. Boletín de la Sociedad Botánica de México 49: 61-69. 
mínimo comparado con la diversidad vegetal existente y el número de plantas que se encuentran en peligro de extinción.

Existen métodos relativamente simples que permiten prolongar la vida de muchas semillas en condiciones de almacenamiento y que pueden llevarse a la práctica de inmediato en conexión con las actividades desarrolladas en herbarios, jardines botánicos, reservas biológicas y otras instituciones similares.

Una parte importante de las especies de semillas ofrecen dificultades con frecuencia insuperables para intentar con ellas medidas de conservación por medio del almacenamiento. A continuación se tratará más ampliamente este problema.

\section{SEMILLAS ORTODOXAS Y RECALCITRANTES}

Hace catorce años Roberts (1973) clasificó a las semillas en dos grupos, de acuerdo a su comportamiento en condiciones de almacén. Una parte importante de las especies vegetales producen semillas cuya viabilidad se prolonga al disminuir su contenido de humedad para almacenarlas a temperaturas bajas. Estas semillas recibieron el nombre de "ortodoxas". En otras especies, especialmente las de semillas grandes, una disminución del contenido de humedad debajo de cierto límite relativamente alto causa la muerte de las semillas y no es posible almacenarlas a baja temperatura. Estas semillas se conocen como "recalcitrantes". En publicaciones posteriores (King y Roberts, 1979; Chin y Roberts, 1980), las características de ambos tipos de semilla han sido descritas con detalle y se han dado listas de especies de uno y otro grupo.

Las peculiaridades de las semillas de cada grupo pueden resumirse de la siguiente manera:

Semillas ortodoxas.- Tienden a ser de talla pequeña, se desprenden de la planta madre con un contenido de humedad generalmente menor del $20 \%$ sobre el peso húmedo. Durante la maduración de estas semillas la gradual deshidratación de sus células conduce a un rearreglo de la estructura macromolecular de manera que se preserva la potencialidad de regeneración de la estructura terciaria de las proteínas cuando las células se hidratan nuevamente. Varios son los mecanismos que se han propuesto para explicar esta resistencia citoplasmática a la deshidratación (Bewley, 1979; Leopold, 1986), una de las fundamentales parece ser el papel que juega el agua unida a macromoléculas, cuya presencia y disposición impide la alteración irreversible de éstas. También la naturaleza de los lípidos de las membranas, las características del contenido vacuolar y otros aspectos de la estructura celular parecen estar relacionados de una u otra manera con la resistencia a la deshidratación.

Cuando las semillas ortodoxas alcanzan bajos niveles de hidratación (menos del 5\% sobre el peso húmedo), su resistencia a las bajas temperaturas se incrementa notablemente, de manera que es posible prolongar la viabilidad en almacenamientos bajo cero grados centígrados. La duración de la viabilidad depende del contenido de humedad y de la temperatura, de lo cual resulta que en algunos casos la longevidad puede prolongarse al doble por cada $5.6 \mathrm{C}$ de disminución de la temperatura y/o por cada $1 \%$ de reducción en el contenido de humedad (Ellis y Roberts, 1980). Seguramente que estos 
parámetros varían en cada especie, ya que los aquí mencionados han sido medidos en gramineas; sin embargo, proporcionan una base para intentar prescribir condiciones de almacenamiento con fines de conservacionismo.

Desde un punto de vista ecológico las semillas ortodoxas son características de aquellas especies para las que en el medio natural es importante la persistencia en el tiempo de las semillas como factor de sobrevivencia. Así encontramos que predominan las especies con semillas ortodoxas entre las plantas anuales y en regiones con estación seca marcada. La mayoría de las plantas cultivadas más importantes presentan este tipo de semillas. En ambientes húmedos las encontramos entre las plantas oportunistas de hábito herbaceo y arbustivo y en pocos árboles.

La gran mayoría de las especies forestales que se utilizan en plantaciones monoespecíficas también las presentan (Wood, 1976; Willam, 1985), como aquellas pertenecientes a los géneros: Pinus, Eucaliptus, Cedrela, Ochroma, Terminalia, Ulmus. El caso de Terminalia ha sido estudiado con detalle (Tompsett, 1986).

Las semillas ortodoxas de las dicotiledoneas tienden a ser epigeas en sentido estricto, muchas poseen cotiledones folíaceos que se vuelven fotosintéticos durante la germinación. Esta puede tener lugar en terreno desnudo bajo amplias fluctuaciones cotidianas de temperatura. Estas semillas por ser pequeñas, pueden absorber agua más fácilmente aún cuando la humedad relativa de la atmósfera, sobre el suelo desnudo, disminuya con el calentamiento diurno.

Semillas recalcitrantes.- En este caso los propágulos maduros generalmente tienden a ser grandes y son liberados con un alto contenido de humedad que llega a representar más del $50 \%$ del peso húmedo de la semilla. Cuando se manejan con el fin de almacenarlas, es imposible hacer descender el contenido de humedad por abajo del $20 \%$ sin causarles daños irreversibles. A diferencia de las semillas ortodoxas, las recalcitrantes matienen un cierto grado de actividad metabólica, por lo que su requerimiento de oxígeno es elevado y mueren al carecer de ventilación adecuada. Ambas características impiden que las semillas puedan sobrevivir a temperaturas bajas y las hacen un sustrato adecuado para el desarrollo de hongos y otros microorganismos que aceleran los daños producidos por el almacenamiento (Christensen, 1978; Chin et al. 1984).

En las semillas recalcitrantes no tienen lugar los drásticos cambios en la disposición de las macromoléculas citoplasmáticas que permiten la deshidratación sin daños en las semillas ortodoxas. En las semillas recalcitrantes la latencia es de una naturaleza más efímera y menos profunda y en muchos casos no se puede considerar que la presenten, pues las semillas tienden a germinar muy rápidamente al diseminarse e incluso se dan casos de viviparidad en los que no hay interrupción clara en el crecimiento del embrión en el periodo que transcurre entre la fecundación y el establecimiento, tal como ocurre en algunos mangles y en ciertas leguminosas.

Las semillas recalcitrantes se presentan principalmente entre las plantas leñosas de ambientes húmedos. Son frecuentes en los árboles de bosques templados caducifolios y en la selva tropical húmeda. Predominan a lo largo del cinturón intertropical, de manera que esta característica es considerada como uno más de los factores que dificultan las medidas de conservación y manejo de las especies en estos ecosistemas $(\mathrm{Ng}, 1976)$.

En la Tabla 1 se presenta una lista de especies de la selva alta perennifolia de Veracruz, México, en la que se observa que el contenido de humedad de las semillas al 


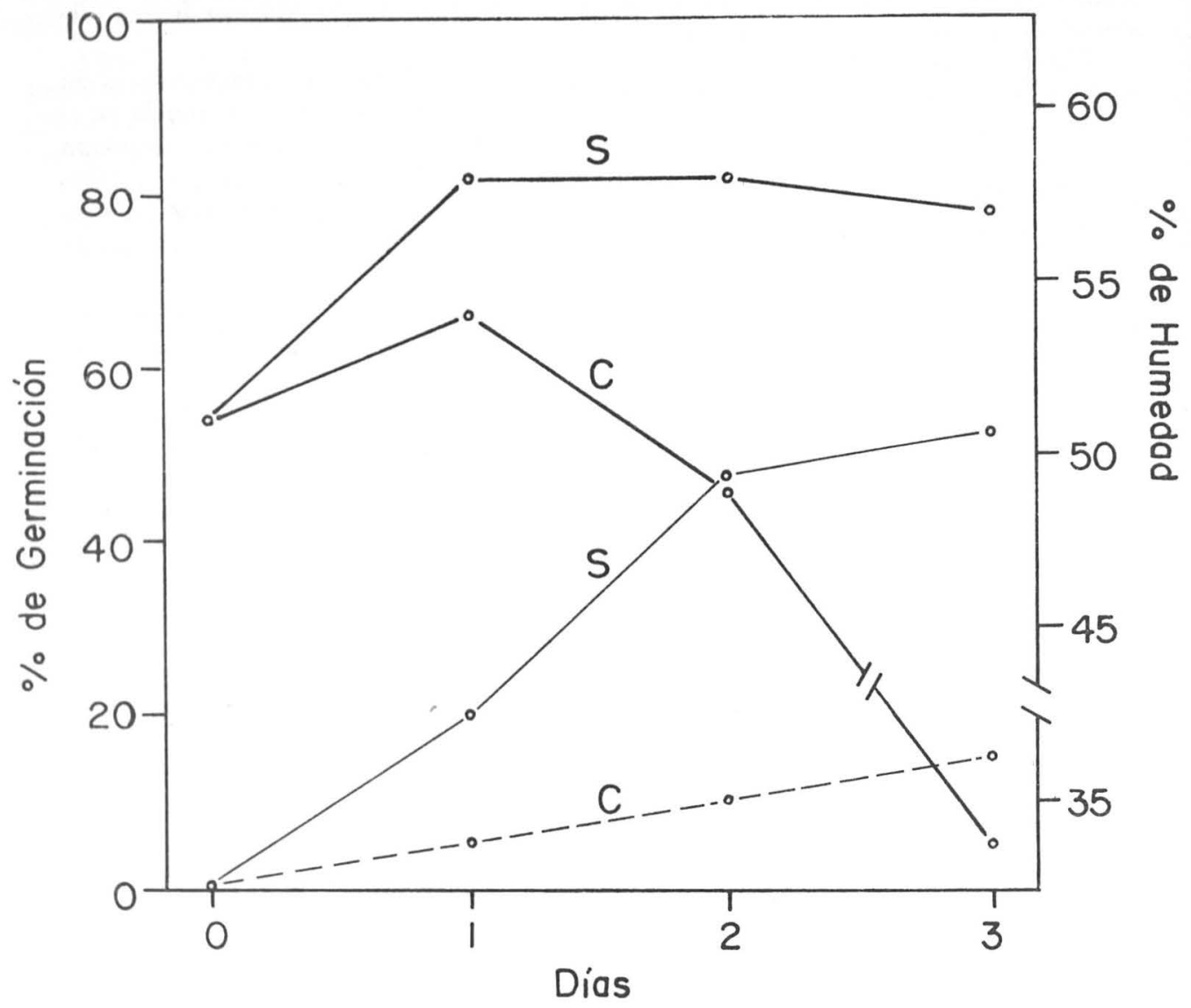

Figura 1.- Cambios en el porcentaje de germinación y el contenido de humedad en tres días, en dos muestras de semillas $(n=40)$ de Pithecellobium arboreum, que fueron colocadas sobre la superficie del suelo de una selva madura (S) y un terreno sin vegetación (C). 1- día nublado y lluvioso, 2, 3 - días soleados. Las semillas germinadas en $\mathrm{C}$ no lograron establecerse. 
Tabla 1.- Contenido de humedad de semillas maduras, libres del fruto, de plantas de la Estación de Biología Tropical de Los Tuxtlas. (*) Indica que esos datos proceden del trabajo de Puchet y Vázquez-Yanes (1987).

\begin{tabular}{|c|c|c|}
\hline ESPECIES & $\begin{array}{l}\text { No. de semillas } \\
\text { usadas en la } \\
\text { determinación }\end{array}$ & $\begin{array}{c}\text { Contenido } \\
\text { de } \\
\text { humedad } \\
(\%)\end{array}$ \\
\hline Astrocaryum mexicanum Liebm. & 11 & 72.74 \\
\hline Pouteria durlandii (Standı.) Baehni & 11 & 72.71 \\
\hline Calatola laevigata Standl. & 10 & 69.28 \\
\hline Heliconia sp. & 20 & 65.24 \\
\hline Siparuna andina (Tul) DC & 40 & 53.94 \\
\hline Nectandra ambigens (Blake)C.K. Allen & * & 51.76 \\
\hline Pithecellobium arboreum (L.) Urban & 10 & 50.87 \\
\hline Pouteria sapota (Jacq.) H.E. Moore et Stearn & 8 & 44.36 \\
\hline Couepia poliandra (H.B.K.) Rose & 10 & 41.20 \\
\hline Chamaedorea tepejilote Liebm. & 20 & 39.25 \\
\hline Calophyllum brasiliense Camb. & 20 & 37.85 \\
\hline Cymbopetallum baillonii R.E. Fries & * & 32.80 \\
\hline \multicolumn{3}{|l|}{ Desmoncus sp 2031.90} \\
\hline Bactris trichophylla Burret Repert & 20 & 26.24 \\
\hline Cecropia obtusifolia Bertol. & 200 & 18.71 \\
\hline Solanum diphyllum $\mathrm{L}$. & 200 & 16.30 \\
\hline Erytrina folkersii Krukoff \& Moldenke & 20 & 8.28 \\
\hline
\end{tabular}


momento de la diseminación es notablemente alto en la mayoría de las especies estudiadas, lo cual indudablemente está indicando que la mayor parte de ellas son recalcitrantes.

Desde el punto de vista de establecimiento en el medio natural, hay una diferencia en el tipo de ambiente en que las semillas recalcitrantes tienden a germinar y establecerse en forma óptima, con respecto a lo que se ha visto para las ortodoxas. Las semillas recalcitrantes se hidratan y germinan mejor en el suelo que se encuentra protegido por vegetación ya establecida, que modera las fluctuaciones cotidianas de temperatura y humedad; en cambio, en suelos desnudos frecuentemente sufren daños que impiden la germinación o el establecimiento de la plántula. Un ejemplo de lo anterior puede verse en la Figura 1, en la que se representa el comportamiento de la germinación y los cambios de contenido de humedad de las semillas de Pithecellobium arboreum colocadas en su medio natural, sobre suelo desnudo y sobre suelo de la selva. A pesar de que este experimento se realizó en plena época lluviosa, con precipitaciones intensas y frecuentes durante el tiempo que duró su seguimiento, es evidente que en el suelo desnudo estas semillas de talla grande y alto contenido inicial de humedad (alrededor del $50 \%$ del peso húmedo), tuvieron desventajas para obsorber y conservar la humedad.

Puesto que las semillas recalcitrantes tienden a ser grandes, producen plántulas grandes. Sus cotiledones suelen ser carnosos, con o sin potencialidad fotosintética, que permanecen en contacto con las semillas hasta agotarse las reservas que contienen. Las plántulas desarrollan una superficie fotosintética relativamente amplia a partir de la reserva de la semilla (Foster y Hansen, 1985; Foster, 1986), lo que les confiere ventajas en ambientes sombreados de bosques y selvas; asimismo raíces más extensas y profundas que pueden representar una ventaja en suelos con intensa competencia por nutrientes

El número de trabajos existentes realizados sobre aspectos de anatomía y fisiología de semillas recalcitrantes es insignificante, comparado con el número tan enorme que existe sobre algunas especies con semillas ortodoxas; no obstante ello, existen buenos trabajos sobre algunas especies de los géneros Araucaria (Tompsett, 1982), Avicennia (Berjak et al.,1984), Dipterocarpus (Tompsett, 1987), Hevea (Chin et al 1981), Theobroma (King y Roberts, 1982), Shorea (Nautiyal y Purohit, 1985a, b, c; Nautiyal et al.1985; Tompsett, 1985), entre varios otros. Estos trabajos destacan la fragilidad de la viabilidad de estas semillas ante la pérdida de humedad.

\section{PRESERVACIÓN DE LA VIABILIDAD EN ALMACÉN}

Para determinar si una semilla va a comportarse, ya sea como ortodoxa o como recalcitrante, al intentar almacenarla, deben de hacerse pruebas de resistencia a la desecación empleando técnicas de deshidratación que no dañen a las semillas por ser demasiado drásticas, lo cual con frecuencia ha conducido a una incorrecta clasificación de las semillas como recalcitrantes cuando en realidad se trataba de semillas ortodoxas. También es importante extremar las precauciones en la colecta y transporte de las semillas antes de esta determinación, ya que factores como calentamiento involuntario, y falta de ventilación durante esta etapa del manejo de las semillas, dan frecuentemente una imagen falsa de sus propiedades. 
Algunos de los métodos de desecación más seguros consisten en el uso de corrientes de aire con bajo contenido de humedad y temperatura relativamente baja (15 5\% de humedad relativa) y $152 \mathrm{C}$ de temperatura), que se hacen incidir sobre monocapas de semillas. Durante el proceso de desecación deben tomarse muestras de semillas con el fin de establecer la relación entre contenido de humedad y viabilidad. Si esta se pierde totalmente a humedades cercanas al $20 \%$ sobre el peso húmedo, casi puede tenerse la certeza de que se trata de semillas recalcitrantes y viceversa, si la humedad desciende del $15 \%$ conservandose la viabilidad, se trata de semillas ortodoxas (Thompsett, 1982, 1985, 1987). Otros métodos de desecación como la liofilización o el uso de desecadores potentes pueden ser demasiado drásticos para algunas especies.

Una vez identificada la naturaleza de las semillas, es posible prescribir, o al menos, ensayar con mayores probabilidades de éxito alguna condición de almacenamiento. Si se trata de una semilla recalcitrante, la posibilidad de un almacenamiento prolongado es muy limitada. Se tendrá que asegurar que las semillas se mantengan con un contenido óptimo relativamente alto de humedad, impidiendo el desarrollo de microorganismos con generosas aplicaciones de fungicidas. Las semillas deberán mantenerse bien ventiladas y a temperaturas arriba del punto de congelación; sin embargo, a pesar de todas las precauciones es poco probable que las semillas puedan mantenerse viables por más tiempo que unos cuantos meses o un año como máximo, ya que los efectos deletéreos de los microorganismos y la alta tasa metabólica pronto conducen al deterioro de las semillas.

Se han investigado procedimientos para prolongar la vida de las semillas recalcitrantes por medio de congelación a muy bajas temperaturas, siendo los resultados hasta ahora bastante ambiguos (Stangood y Bass, 1981). Resta aún mucho por hacer en este campo (Vázquez-Yanes, 1987).

Las semillas ortodoxas pueden almacenarse por años o siglos a baja temperatura. De acuerdo con Chin y Roberts (1980), las semillas ortodoxas almacenadas en contenedores cerrados, con un contenido de humedad residual del 5 al $7 \%$ a $18 \mathrm{C}$ de temperatura o menos, teóricamente podrían conservarse viables por un siglo o más sin una disminución significativa de la viabilidad. Algunas semillas ortodoxas han sobrevivido a temperaturas que se acercan al cero absoluto sin daño.

Un método que ha probado ser efectivo para la conservación de las semillas pequeñas es el siguiente: 1 ) desecar las semillas frescas con una corriente de aire de baja humedad, hasta alcanzar una humedad en las semillas de aproximadamente el 5\%; 2) colocar las semillas en viales de vidrio y poner sobre ellas un tapón de gasa u algodón no muy comprimido; 3) terminar de llenar el vial con una sustancia deshidratante como el gel de sílice y sellar la boca del vial con fuego. 4) guardar los viales en refrigeradores a temperaturas de -10 a $-20 \mathrm{C}$, procurando que ésta se mantenga estable.

Con el procedimiento anterior es posible asegurar la conservación del germoplasma de plantas que producen semillas ortodoxas y además contar con material vivo para estudios, para propagación o para intercambio. 


\section{CONCLUSIONES}

Como hemos visto en las líneas anteriores, el almacenamiento de semillas con la finalidad de conservar el germoplasma puede realizarse con grandes posibilidades de éxito en especies que producen semillas ortodoxas; en cambio, las perspectivas al emplear semillas recalcitrantes son bastante desalentadoras para muchas especies. Se requiere profundizar en el conocimiento biológico de las semillas para encontrar medios adecuados que permitan el mantenimiento de su viabilidad por largo tiempo. En las especies que poseen muy altos contenidos de humedad al momento de la diseminación, las probabilidades de lograr esto son remotas. Es posible que a través del uso de algunas técnicas de ultracongelación y combinadas con el uso de crioprotectores y otros métodos similares puede lograrse progresar en este campo.

Una medida relativamente sencilla de llevar a la práctica es el establecimiento de bancos para el almacenamiento y conservación de semillas ortodoxas en instituciones que se interesan por el estudio de plantas silvestres, para ello existen métodos y procedimientos que han probado su efectividad en bancos de semillas de varios países del mundo.

\section{LITERATURA CITADA}

BERJAK, P., M. DINI y N. W. PAMMENTER. 1984. Possible mechanism underlying the differing dehydratation responses in recalcitrant and ortodox seeds: desication- associated subcellular changes propagules of Avicenia marina. Seed Sci. \& Technol. 12: 365-384.

BEWLEY, J. D. 1979. Physiological aspects of desiccation tolerance. Ann. Rev. Plant Physiol. 30: 195-238. CHIN, H. F. y E. H. ROBERTS. 1980. Recalcitrant crop seeds. Tropical Press, Malaysia.

CHIN, H. F., B. AZIZ, B. B. ANG y S. HAMZAH. 1981. The effect of moisture and temperature on the ultraestructure and viavility of Hevea brasiliensis. Seed Sci. \& Technol. 9: 411-422.

CHIN, H. F. y L. HOR. y M. B. MOHD LASSIM. 1984. Identification of recalcitrant seeds. Seed Sci. \& Technol. 12: 429-436.

CHRISTENSEN, C. M. 1978. Moisture and seed decay. En: Koslowski, T. T. (Ed.) Water deficits and plant growth. p.p. 199-219. Academic Press, New York.

ELLIS, R. H. y E. H. ROBERTS. 1980. The influence of temperature and moisture on seed viavility period in barley Hordeum distichum L.) Ann. Bot. (London) 45: 31-37.

FOSTER, S. A. 1986. On the adaptative value of large seeds for tropical moist forest: A review and synthesis. Bot. Rev. 52: 260-299.

FOSTER, S. A. y C. H. JANSON. 1985. The relationship between seed size and establishment conditions in tropical wood plants. Ecology 66: 773-720.

KING, M. W. y E. H. ROBERTS. 1979. The storage of recalcitrant seeds. achievements and possible approaches. Report for the International Board for Plant Genetic Resources. FAO. Rome.

KING, M. W. y E. H. ROBERTS. 1982. The imbibided storage of cocoa (Theobroma cacao). Seed Sci. \& Technol. 10: 535-540.

LEOPOLD, C. (Editor). 1986. Membranes, metabolism and dry organisms. Comstock Pub. London. 


\section{SEMILLAS}

NAUTTYAL, A. R. y A. N. PUROHIT. 1985a. Seed viability in sal. I. Physiological and biochemical aspects of seed development in Shorea robusta. Seed Sci. \& Technol. 13: 59-68.

NAUTTYAL, A. R. y A. N. PUROHIT. 1985b. Seed viability in sal II. Phisiological and biochemical aspects of ageing in seeds of Shorea robusta. Seed Sci. \& Technol. 13: 69-76.

NAUTTYAL, A. R. y A. N. PUROHIT. 1985c. Seed viability in sal III. Membrane disruption in ageing seeds of Shorea robusta. Seed Sci. \& Technol. 137: 77-82.

NAUTIYAL, A. R. y A. N. PUROHIT. 1985d. Seed viability in sal IV. Protein changes accompanying loss of viability in Shorea robusta. Seed Sci. \& Technol. 13: 83-86.

NG, F. S. P. 1976. The problems of forest tree seed production with reference to dipterocarps. pp. 181-186 En: Chin, H. F., I. C. Enoch y R. M. Raja (Edrs.). Seed technology in the tropics. University Pertanian, Malaysia.

POUCHET, C. E. y C. VAZQUEZ-YANES. 1987. Heteromorfismo críptico en las semillas recalcitrantes de tres especies arbóreas de la selva tropical húmeda de Veracruz, México. Phytologia 62: 100-106.

ROBERTS, E. H. 1973. Predicting the storage life of seeds. Seed Sci. \& Technol. 1: 499-514.

Stanwood, P. C. y L. N. Bass. 1981. Seed germplasm preservation using liquid nitrogen. Seed Sci. \& Technol. 9: 423-437.

TOMPSETT, P. B. 1982. The effect of desiccation on the longevity of seeds of Araucaria hunsteinii and $A$. cunninghamii. Ann. Bot. (London) 50: 639-704.

TOMPSETT, P. B. 1985. The influence of moisture content and storage temperature on the viability of Shorea almon, Shorea robusta, and Shorea roxburgii seed. Canad. J. For. Res. 15: 1074-1079.

TOMPSETT, P. B. 1986. The effect of temperature and moisture content on the longevity of seed of Ulmus carpinifolia and Terminalia brassii. Ann. Bot. 57: 875-883.

TOMPSETT, P. B. 1987. Desiccation and storage studies on Dipterocarpus seeds. Ann. Appl. Biol. 110: 371-379.

VAZQUEZ-YANES, C. 1987. El papel del banco de semillas en la conservación de especies vegetales. Ciencia 38: 239-246.

WILLIAN, R. L. 1985. A guide to forest seed handling with special reference to the tropics. Danida-FAO Forestry Paper, Rome 20(2): 1-279.

WOOD, P. J. 1976. The development of tropical plantations and the need for seed and genetic conservation. pp. 11-18. En: Burley J. y B. T. Styles (Edrs.) Tropical trees variation. Breeding and conservation. p. 11-18. Linnean Society Symposium Serie 2, Academic Press, London. 